\section{DNA variation in myoMIRs of the 1, 133, and 208 families in hypertrophic cardiomyopathy}

\author{
María Palacín, ${ }^{1}$ Eliecer Coto, ${ }^{1,3,4}$ \\ Julián R. Reguero, ${ }^{2}$ María Martín, ${ }^{2}$ \\ César Morís, ${ }^{2,3}$ Belén Alonso, ${ }^{1}$ \\ Marta Díaz, ${ }^{1}$ Ana I. Corao, \\ Victoria Alvarez ${ }^{1}$
}

${ }^{1}$ Genética Molecular, Hospital Central

Asturias; ${ }^{2}$ Cardiología-Fundación

Asturcor, Hospital Universitario Central

Asturias; ${ }^{3}$ Departamento Medicina,

Universidad Oviedo, Oviedo;

${ }^{4}$ Red de Investigación Renal-REDINREN;

${ }^{4}$ Instituto Reina Sofía de Investigación

Nefrológica, Spain

\section{Abstract}

MicroRNAs (miRNAs) are small RNAs that bind to mRNAs and regulate gene expression. MyoMirs are miRNAs implicated in cardiogenesis. Some MyoMirs have been found deregulated in hearts from patients with left ventricular hypertrophy (LVH). DNA variants at these miRNAs could contribute to the risk of developing hypertrophic cardiomyopathy (HCM). To test this hypothesis we used single strand conformation analysis and direct sequencing to search for DNA variants in the mir-208a, miR-208b, miR-133a-1, miR-133a-2, miR-133b, miR-1-1, and miR-1-2 genes in patients with HCM $(n=245)$, LVH secondary to hypertension $(\mathrm{n}=120)$, and healthy controls $(n=250)$. We found several nucleotide variants. Genotyping of patients and healthy controls showed significantly associations between a 133a-1 polymorphism and HCM and a 133b polymorphism and hypertensive$\mathrm{LVH}$. We concluded that rare variants in these mature miRNAs would be rarely found among HCM patients, but miR-133a-1 and 133b polymorphisms could contribute to the risk of developing cardiac hypertrophy.

\section{Introduction}

Micro RNAs (miRNAs) are small non-coding RNAs that negatively regulate gene expression at the posttranscriptional level. By base pairing to the 3' untranslated regions (3'UTRs) of messenger RNAs (mRNAs), miRNAs repress the expression of many genes and regulate physiological processes such as cell differentiation, growth, proliferation, and apoptosis. ${ }^{1}$ Several miRNAs play an important role in myo- genesis and heart development and function, and are designated as MyoMIRS.

The biogenesis of miRNAs involves several steps: first a pri-miRNA is synthesized by the action of RNA polymerase II; in a second step, the pri-miRNA is processed by the enzyme Drosha to give an approximately 90 nucleotides long pre-miRNA. ${ }^{2}$ Pre-miRNAs form several stem-loop secondary structures that are necessary for a correct cleavage that renders a double stranded miRNA, that is then processed to the single-strand mature miRNA and incorporated into the RNA induced silencing complex (RISC; a ribonucleoprotein complex that regulates mRNA expression). ${ }^{3}$ The genes encoding miRNAs are mono- or polycystronic (i.e. the miR-1-1/miR-133a-2 and miR-1-2/miR-133a-1 clusters), and either inserted in introns (i.e. miR208a and 208b) or in intergenic regions. A genome-wide survey of human single nucleotide polymorphisms (SNPs) suggested that $<1 \%$ of the miRNAs have SNPs in their mature sequences, while $\sim 10 \%$ of the premiRNA sequences are polymorphic. ${ }^{4}$ These premiRNA polymorphisms could affect the processing of miRNAs, being thus of relevance for the susceptibility to diseases. ${ }^{5}$

Left ventricular hypertrophy (LVH) is a pathological response to some cardiovascular diseases, such as valvular disease, ischemic heart disease, and hypertension. Hypertrophic cardiomyopathy (HCM) is the left ventricular wall thickening in the absence of cardiac or systemic disease capable of producing the hypertrophy. HCM is frequently familial and transmitted as an autosomal-dominant trait, and the genes primarily involved in HCM encode proteins of the sarcomere. ${ }^{6}$ However, in all the large reported cohorts a significant number of patients did not have sarcomeric mutations, suggesting that other not yet identified genes could be implicated in this disease. ${ }^{7}$ Recent reports have elucidated the role of several miRNAs in cardiac hypertrophy. ${ }^{8}$ The overexpression of some miRNAs (i.e. miR-208) has been linked to hypertrophy in mice. ${ }^{9}$ In contrast, in vitro overexpression of miR-133 and miR-1 inhibited cardiac hypertrophy, while suppression of the miR-133 pathway induced hypertrophy. ${ }^{10}$ These miRNAs enhanced myoblast proliferation by repressing the expression of serum response factor, an essential activator of myogenesis, and several genes that have been implicated in cardiogenesis and hypertrophy are targets of miR-133. ${ }^{8,11}$ The expression of miR-133 has been assessed in murine models of cardiac hypertrophy. The transverse aortic archconstricted mice developed pressure overload and cardiac myocyte hypertrophy and showed a reduced expression of miR-133, indicating that this miRNA family could be involved in the development of cardiac hypertrophy in response to hypertension. ${ }^{11}$

Based on the role of several miRNAs in the
Correspondence: Eliecer Coto, Genética Molecular, HUCA-Maternidad 33006-OviedoSpain. E-mail: eliecer.coto@sespa.princast.es

Key words: microRNAs, myoMIRs, cardiac hypertrophy, DNA polymorphisms, genetic association.

Acknowledgements: this work was supported by grants from the Spanish Fondo de Investigaciones Sanitarias-Fondos FEDER European Union (FIS-00/0172), and Red de Investigación Renal-REDINREN (RD06/0016). MP a predoctoral fellow from FICYT-Principado de Asturias. MD is the recipient of a Contrato de Apoyo a la Investigación- Fondo de Investigaciones Sanitarias.

Received for publication: 1 September 2011.

Revision received: 25 October 2011.

Accepted for publication: 26 October 2011

This work is licensed under a Creative Commons Attribution NonCommercial 3.0 License (CC BYNC 3.0).

(O) Copyright M. Palacín et al., 2011

Licensee PAGEPress, Italy

Cardiogenetics 2011; 1:e12

doi:10.4081/cardiogenetics.2011.e12

pathogenesis of HCM and the association between miRNA gene variants and some diseases, we hypothesised that myoMIR variants could also act as HCM-risk factors. To test this hypothesis we defined the DNA variation at several miRNAs in a group of HCM-patients and healthy controls.

\section{Materials and Methods}

\section{Patients and controls}

The study was based on a total of $245 \mathrm{HCM}$ patients recruited through the Cardiology Department of HUCA.

The inclusion criteria and main characteristics of these patients had been previously reported. ${ }^{12,13}$ HCM was diagnosed based on the echocardiographic finding of a left ventricular septum $>15 \mathrm{~mm}$ in the absence of any other disease that could explain the hypertrophy. All the HCM patients were screened for mutations in the most frequently mutated sarcomeric genes: beta myosin heavy chain (MYH7), cardiac troponin T (TNNT2), alphatropomyosin (TPM1), cardiac troponin I (TNNI3), and myosin binding protein C3 (MYBPC3). ${ }^{12}$ We also studied 120 patients with LVH secondary to hypertension. The control group consisted on 250 healthy individuals who did not have symptoms of cardiac hypertrophy. However, they were not echocardiographically examined to exclude the exis- 
tence of asymptomatic LVH. Patients and controls were Caucasians from the same region (Asturias, Northern Spain, total population 1 million), and gave their informed consent to participate in the study, approved by the Ethical Committee of Hospital Central Asturias.

\section{Identification of myoMIR variants}

All the experimental procedures are available upon request to the corresponding author. Genomic DNA was obtained from $10 \mathrm{~mL}$ of blood, and 350 to 450 nucleotides of the mir208a, -208b, -133a-1, -133a-2, -133b, -1-1, and 1-2 genes were polymerase chain reaction (PCR)-amplified. To search for sequence variants, the PCR fragments were amplified from all the HCM patients and analysed through single strand conformation analysis (SSCA). PCRfragments from individuals who showed heterogeneous SSCA patterns were sequenced using BigDye chemistry in an automated ABI3130 capillary system (Applied Biosystems, Foster City, CA, USA) to identify the nucleotide changes. The nucleotide variants were numbered according to the mature miRNA sequences, with -1 and +1 as the first 5 ' upstream and 3' downstream nucleotide, respectively.

\section{Genotyping of myoMIR variants}

All the patients and controls were genotyped for the myoMIR variants based on their SSCA patterns (208a, 208b, 133a2) or through digestion with a restriction enzyme (PCR-RFLP; $133 \mathrm{~b}$ and $133 \mathrm{al}$ ) as reported. ${ }^{14}$ To validate the accuracy of these methods we confirmed the genotype from several individuals by sequencing the PCR-fragments (Figure 1).

\section{Statistical analysis}

Allele and genotype frequencies in patients and controls were compared through a chisquared test. Odds ratios (OR) with $95 \%$ confidence intervals (CI) were obtained to calculate the relative risk associated with the genotypes. Because sarcomeric mutations can explain the development of HCM in some patients, in order to maximize the chance of detecting any effect MyoMIR polymorphisms on HCM-risk we also compared the allele/genotype frequencies between patients with and without sarcomeric mutations and controls. The chi-squared was also used to analyse the deviation from the Hardy-Weinberg equilibrium of the genotype frequencies. All statistical analyses were performed with the SPSS statistical package (v.11.0).

\section{Results}

Table 1 summarizes the main characteris-

tics of patients and controls. SSCA of the 10 myoMIRs in the HCM patients showed heterogeneous electrophoretic patterns for the PCR fragments of 208a, 208b, 133a-1, 133a-2, and $133 \mathrm{~b}$. A total of seven nucleotide changes were identified, and two were new changes (no reported as polymorphisms in the ensembl database; www.ensembl.org). Allele and genotype frequencies for these variants in patients

and controls are summarised in Table 2.

We found two nucleotide variants in the miR-133a-1 and 133b sequences. Carriers of the miR-133a- $1+85 \mathrm{~A}$ were significantly more frequent in HCM patients without sarcomeric mutations (12\%) compared to controls (5\%; $\mathrm{P}=0.02, \quad 0 \mathrm{R}=2.30, \quad 95 \%=1.13-4.67), \quad \mathrm{HCM}$ patients with sarcomeric mutations, and LVHhypertensives. Carriers of the -90A-deletion in

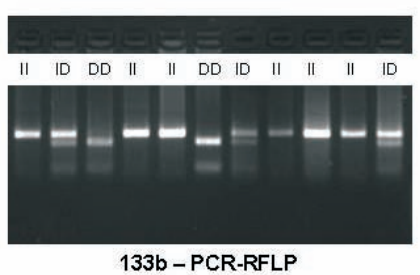

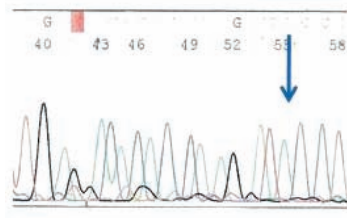

II

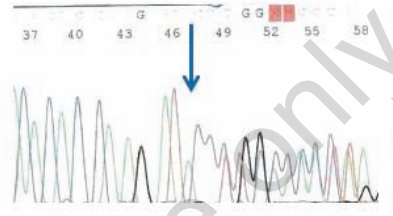

ID

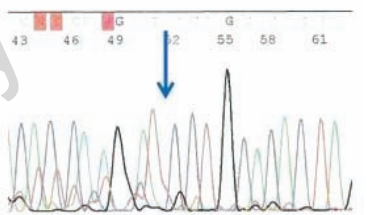

$\mathrm{DD}$

Figure 1. Single strand conformation analysis (SSCA) and polymerase chain reaction (PCR)-RFLP patterns and sequence for the three $133 \mathrm{~b}$ ins/del A genotypes.

Table 1. Main characteristics of the patients with HCM and LVH secondary to hypertension.

\begin{tabular}{lcc} 
& HCM $(n=245)$ & Hypertensive LVH $(n=120)$ \\
Mean age at diagnosis (years) & $45 \pm 13$ & $58 \pm 17$ \\
Rang & $18-75$ & $35-75$ \\
Male & $144(59 \%)$ & $71(59 \%)$ \\
\hline
\end{tabular}

\section{Family history} (HCM or sudden death)

\section{Mean BMI}

Male

Female

Mean IVS (mm)

Mean PWT (mm)

Mean LVWT

Dyspnea

NYHA index

Class I-II

Class III-IV

Angina

Syncope

Atrial fibrillation

Arrhythmia (Holter monitoring)

LVOT $>30 \mathrm{~mm} \mathrm{Hg}$

\section{Sarcomeric mutations}

MYH7

MYBPC3

TNNT2

TPM1

TNNI3

HCM, hypertrophic cardiomyopathy; LVH, left ventricular hypertrophy; IVS, interventricular septum; PWT, posterior wall thic
ventricular wall thickness; NYHA, New York Heart Association functional class; LVOT, left ventricular outflow tract gradient.
$105(43 \%)$

$\begin{array}{ll}27 \pm 3 & 28 \pm 4 \\ 26 \pm 4 & 28 \pm 5 \\ 20 \pm 5 & 15 \pm 5 \\ 13 \pm 5 & 10 \pm 6 \\ 34 \pm 6 & 26 \pm 6\end{array}$

$168(69 \%) \quad 36(30 \%)$

$120(48 \%) \quad 28(23 \%)$

$48(20 \%) \quad 8(7 \%)$

$96(39 \%) \quad 19(16 \%)$

$48(20 \%) \quad 7(6 \%)$

$47(19 \%) \quad 18(15 \%)$

$55(22 \%) \quad 20(17 \%)$

$72(29 \%) \quad 32(27 \%)$

$40(16 \%)$

$12(5 \%)$

$22(9 \%)$

$4(2 \%)$

$1(<1 \%)$

, int OPEN $\bigcirc$ ACCESS 
miR-133b were significantly more frequent among the LVH-hypertensives (17\%) compared to controls ( $8 \% ; \quad \mathrm{P}=0.03, \quad \mathrm{OR}=1.99,95 \%$ $\mathrm{CI}=1.06-3.76$ ), and HCM patients (Table 2). Moreover, two LVH-hypertensives were delA homozygotes compared to none in the control and HCM groups. This suggested that the -90 del A could be a risk factor for LVH secondary to hypertension. An online analysis (MFold, http://mfold.rna.albany.edu) showed that the miR-133a-1 and miR-133b polymorphisms altered the secondary structure of the two primiRNAs (Figure 2).

We found three previously reported $133 \mathrm{a}-2$ SNPs: -191 G/A (SNP reference number rs45547937), 171G/A (rs13040566), and -88 G/A (rs13040413). Sequencing of several cases with different SSCA patterns showed three different allele combinations: -191AA/-171AA/88AA, -191GG/-171GG/-88GG, and -191AG/$171 \mathrm{AG} /-88 \mathrm{AG}$. This suggested that the three 133a-2 SNPs were in complete linkage disequilibrium, defining two haplotypes: -191A/-171A/88A and -191G/-171G/-88G. No significant difference was found between patients and controls for these polymorphisms.

We found a rare miR-208a variant (-175 $\mathrm{C}>\mathrm{A}$ ) in one HCM patient, a 66 years old female with a septum of $26 \mathrm{~mm}$ and no mutations in the sarcomeric genes. No relatives were available for the genetic study to determine whether this variant segregated with the disease.

\section{Discussion}

The variation at the mature miRNA sequences could affect the binding of their target mRNAs (the seed region) and affect the capacity to regulate gene expression. Naturally occurring changes in these critical miRNA nucleotides are very rare. In a search for nucleotide variants in 227 human miRNAs, Duan et al. only found one SNP in the mature miR-125a. ${ }^{5}$ Nucleotide changes in mature miRNAs are commonly found in tumor tissues as acquired mutations associated with the development of several cancer types. ${ }^{15-17}$ None of the myoMIR variants found in our study was in the mature miRNA sequences. We thus concluded that nucleotide changes in these critical regions would not contribute to the risk of developing cardiac hypertrophy.

We found a significant association between common variants in the $133 \mathrm{a}-1$ and $133 \mathrm{~b}$ miRNA genes and HCM without sarcomeric mutations and LVH secondary to hypertension, respectively. Nucleotide variants in miRNA genes could disrupt the normal RNA secondary structure and could have an effect on disease risk through the processing and expression of the pre-miRNA and mature miRNA. ${ }^{16}$ All the

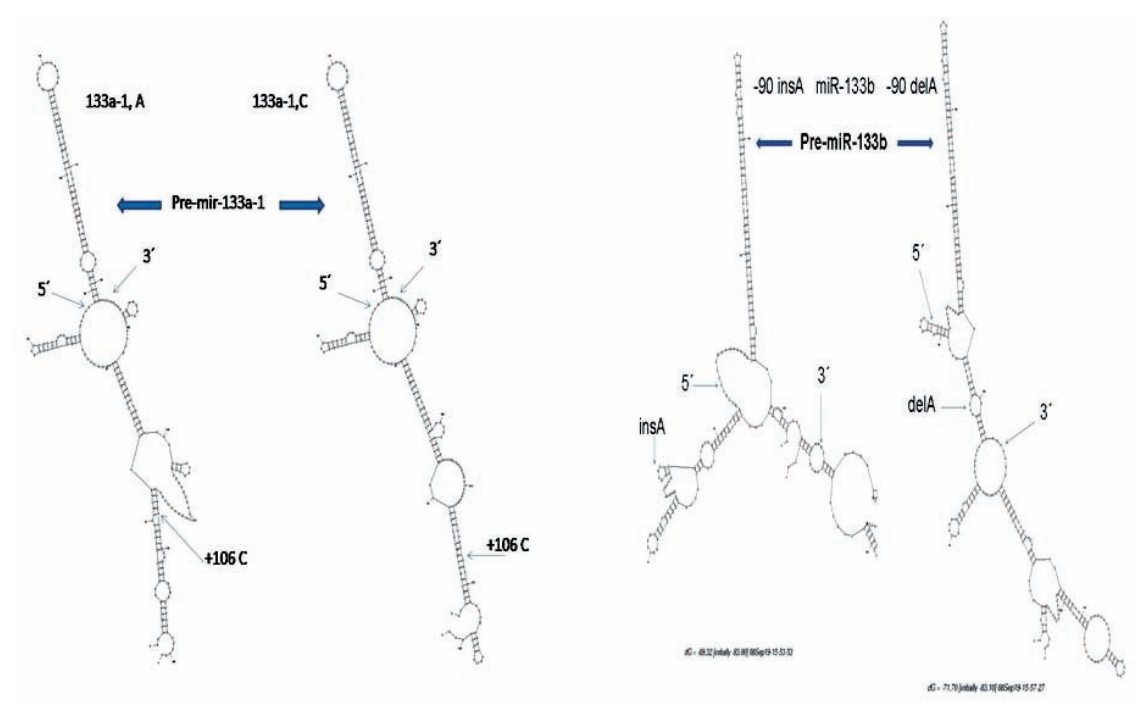

Figure 2. Secondary structure of the $133 \mathrm{~A}-1$ and $133 \mathrm{~b}$ pri-miRNAs containing the two alleles. The 5' and 3' ends of the pre-miRNAs are also indicated.

Table 2. Genotype and allele frequencies for the myoMIR polymorphisms.

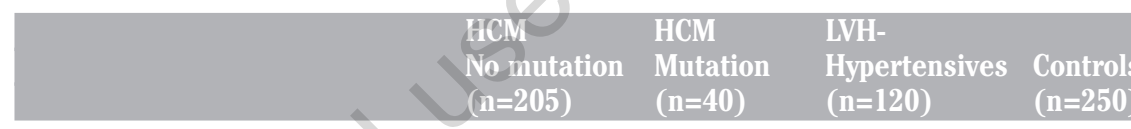

$\operatorname{miR}-133 a-1^{*}$

$+85 \mathrm{~A} / \mathrm{C}$

$\begin{array}{lcccc}\mathrm{AA} & 3(2 \%) & 0 & 0 & 0 \\ \mathrm{AC} & 20(10 \%) & 2(5 \%) & 4(3 \%) & 13(5 \%) \\ \mathrm{CC} & 182(88 \%) & 38(95 \%) & 116(97 \%) & 237(95 \%) \\ \mathrm{A} & 0.06 & 0.03 & 0.02 & 0.03 \\ \mathrm{C} & 0.94 & 0.97 & 0.98 & 0.97 \\ \text { miR-133a-2: } & & & \\ \text {-191G/A-171G/A-88G/A } & & & \\ \text { (rs45547937/rs13040566/rs13040413) } & 8(4 \%) & 2(5 \%) & 7(6 \%) & 10(4 \%) \\ \text { AA/AA/AA } & 65(32 \%) & 12(30 \%) & 41(34 \%) & 82(33 \%) \\ \text { AG/AG/AG } & 131(64 \%) & 26(65 \%) & 72(60 \%) & 158(63 \%) \\ \text { GG/GG/GG } & 0.20 & 0.20 & 0.23 & 0.18 \\ \text { AAA } & 0.80 & 0.80 & 0.77 & 0.82 \\ \text { GGG } & & & \end{array}$

$\operatorname{miR}-133 b^{* *}$ :

-90 ins/delA

$\begin{array}{llll}\text { ins/ins } & 187(91 \%) & 37(92 \%) & 99(83 \%)\end{array} 230(92 \%)$

ins/de

del/del

insA

delA

$18(9 \%)$

0

0.96

0.04

$\begin{array}{cc}3(8 \%) & 18(15 \%) \\ 0 & 3(2 \%) \\ 0.96 & 0.90 \\ 0.04 & 0.10\end{array}$

$19(8 \%)$

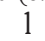

0.95

0.05

miR-208 a.

-175 C/A (rs28730766)

$\begin{array}{lcccc}\mathrm{CC} & 204(99 \%) & 40 & 120 & 250 \\ \mathrm{CA} & 1(1 \%) & 0 & 0 & 0 \\ \mathrm{AA} & 0 & 0 & 0 & 0\end{array}$

miR-208 b:

+95 C/T (rs45489294)

$\mathrm{CC}$

CT

TT

C

$\begin{array}{cccc}200(98 \%) & 38(95 \%) & 116(97 \%) & 244(98 \%) \\ 5(2 \%) & 2(5 \%) & 4(3 \%) & 6(2 \%) \\ 0 & 0 & 0 & 0 \\ 0.99 & 0.97 & 0.98 & 0.90 \\ 0.01 & 0.03 & 0.02 & 0.10\end{array}$

HCM, hypertrophic cardiomyopathy; LV, left ventricular; LVH, left ventricular hypertrophy. * $\mathrm{P}=0.02, \mathrm{OR}=2.30$ (1.13-4.67), $\mathrm{AA}+\mathrm{AC}$ vs. CC, $\mathrm{HCM}$ no mutation us. controls, $\mathrm{P}=0.006, \mathrm{OR}=2.53$ (1.28-5.01) A vs. C, HCM no mutation us. controls; ${ }^{*} \mathrm{P}=0.03, \mathrm{OR}=1.99(1.06-3.76$, del/del + ins/del vs. ins/ins, LVH us. controls, $\mathrm{P}=0.01, \mathrm{OR}=2.11$ (1.18-3.78) ins $v$ s. del, LVH $v$ s. controls. 
myoMIR variants found in our study were out of the pre-miRNA sequence and a direct effect on the secondary structure of the doublestranded stem of the pre-miRNA hairpin could thus be excluded. Although the 133a-1 and 133b nucleotide polymorphisms would alter the pri-miRNAs secondary structure, structurally relevant changes in pri-miRNAs would not affect processing. ${ }^{18}$

These nucleotide changes could modify the binding of transcription factors (TFs) and enhancers, regulating miRNA gene expression. Mice lacking the myocyte enhancer factor-2 (MEF2) exhibited a down-regulation of the miR-1-1/miR-133a-2 and miR-1-2/miR133a-1 transcripts, and a MEF2 enhancer sequence located between miR-1-1 and 133a-2 has been identified. ${ }^{10}$ The mutation of this MEF2 enhancer abolished miR-1-1/133a-2 expression on cardiac and skeletal muscle cells of transgenic mice. A MEF2-like site was also identified in the region between miR-1-1 and miR-133a-2, suggesting that the two miR$1 /$ miR-133a clusters contain similar regulatory elements. ${ }^{19}$ We searched for putative TFs binding sites in the polymorphic sites found in our study. According to the TFSEARCH database (http://mbs.cbrc.jp/research/db/TFSEARCH.htm l), the 133a-1 SNP would not create/destroy binding sites for known TFs. However, the -90 ins/del A in 133b affected a binding site for TFs of the GATA family, that have been implicated in cardiogenesis and cardiac hypertrophy. ${ }^{20,21}$ The -90 del A should destroy a binding site for these TFs, and could thus reduce the expression of miR-133b, that in turn could have a prohypertrophic effect. Functional studies are necessary to confirm this putative effect of the $133 \mathrm{~b}$ ins/del on gene expression.

The absence of a significant effect of these miRNA variants on HCM with sarcomeric mutations likely reflects the fact that in these patients the disease was caused by these mutations. However, we cannot exclude an effect of these polymorphisms as modifiers of disease severity and adverse events among HCM patients with sarcomeric mutations. The higher frequency of the 133b polymorphism among the LVH hypertensives could be explained by a role of this miRNA in the regulation of physiological pathways that lead to the development of LVH in response to hypertension. ${ }^{22}$ Alternativelly, this polymorphism could be related with the risk of developing hypertension itself. A comparison between -90 delA frequencies in large cohorts of hypertensives vs. normotensives should be necessary to clarify this.

We are well aware that our work has several limitations. First, SSCA is an indirect technique for detecting nucleotide changes in PCR-fragments. Although the rate for false negatives is very low $(<5 \%)$ we can not exclude that some nucleotide variants in the myoMIRs were not identified in our study. Second, the association of the genotypes with the risk of cardiac hypertrophy was based on a limited number of patients and from a single population. The replication of our study with larger series of patients/controls and from different populations is necessary to confirm / refute our results. In addition, functional studies should be necessary to determine the effect of the nucleotide variants on miRNAs maturation and expression.

\section{Conclusions}

We reported nucleotide variants in the sequences of miR-1, 133, and 208 genes. A miR-133a-1 SNP could be a risk factor for HCM, while a miR-133b polymorphism was associated with cardiac hypertrophy secondary to hypertension. To our knowledge, this is the first report that described the association between microRNA gene variants and cardiac hypertrophy.

\section{References}

1. Valencia-Sánchez MA, Liu J, Hannon GJ, Parker R. Control of translation and mRNA degradation by miRNAs and siRNAs. Genes Dev 2006;20:515-24.

2. Lee $\mathrm{Y}$, Kim M, Han J, et al. MicroRNA genes are transcribed by RNA polymerase II. Embo J 2004;23:4051-60.

3. Bartel DP. MicroRNAs: genomics, biogenesis, mechanism, and function. Cell 2004;116:281-97.

4. Saunders MA, Liang H, Li WH. Human polymorphism at microRNAs and microRNA target sites. Proc Natl Acad Sci U S A 2007;104:3300-5.

5. Duan R, Pak C, Jin P. Single nucleotide polymorphism associated with mature miR-125a alters the processing of primiRNA. Hum Mol Genet 2007;16:1124-31.

6. Arad M, Seidman JG,Seidman CE. Phenotypic diversity in hypertrophic cardiomyopathy. Hum Mol Genet 2002;11: 2499-506.

7. Friddle CJ, Koga T, Rubin EM, Bristow J. Expression profiling reveals distinct sets of genes altered during induction and regression of cardiac hypertrophy. Proc Natl Acad Sci U S A 2000;97:6745-50.

8. van Rooij E, Sutherland LB, Liu N, et al. A signature pattern of stress-responsive microRNAs that can evoke cardiac hyper- trophy and heart failure. Proc Natl Acad Sci U S A 2006;103:18255-60.

9. van Rooij E, Sutherland LB, Qi X, et al. Control of stress-dependent cardiac growth and gene expression by a microRNA. Science 2007;316:575-9.

10. Liu N, Williams AH, Kim Y, et al. An intragenic MEF2-dependent enhancer directs muscle-specific expression of microRNAs 1 and 133. Proc Natl Acad Sci USA 2007;104:20844-9.

11. Care A, Catalucci D, Felicetti F, et al. MicroRNA-133 controls cardiac hypertrophy. Nat Med 2007;13:613-8.

12. García-Castro M, Coto E, Reguero JR, et al. Mutations in sarcomeric genes MYH7, MYBPC3, TNNT2, TNNI3, and TPM1 in patients with hypertrophic cardiomyopathy. Rev Esp Cardiol 2009;62:48-56.

13. Coto E, Palacín M, Martín M, et al. Functional polymorphisms in genes of the Angiotensin and Serotonin systems and risk of hypertrophic cardiomyopathy: AT1R as a potential modifier. $\mathrm{J}$ Trans Med 2010;8:e64.

14. de Mena L, Coto E, Cardo LF, et al. Analysis of the Micro-RNA-133 and PITX3 genes in Parkinson's disease. Am J Med Genet B Neuropsychiatr Genet 2010;153B:1234-9.

15. Hu Z, Chen J, Tian T, et al. Genetic variants of miRNA sequences and non-small cell lung cancer survival. J Clin Invest 2008;118:2600-8.

16. Jazdzewski K, Murray EL, Franssila K, et al. Common SNP in pre-miR-146a decreases mature miR expression and predisposes to papillary thyroid carcinoma. Proc Natl Acad Sci U S A 2008;105:7269-74.

17. Shen J, Ambrosone CB, DiCioccio RA, et al. A functional polymorphism in the miR146a gene and age of familial breast/ovarian cancer diagnosis. Carcinogenesis 2008;29:1963-6.

18. Diederichs S, Haber DA. Sequence variations of MicroRNAs in human cancer: alterations in predicted Secondary Structure do not Affect processing. Cancer Res 2006;66:6097-104.

19. Zhao Y, Samal E, Srivastava D. Serum response factor regulates a muscle-specific microRNA that targets Hand2 during cardiogenesis. Nature 2005;436:214-20.

20. Akazawa H, Komuro I. Roles of cardiac transcription factors in cardiac hypertrophy. Circ Res 2003;92:1079-88.

21. Pikkarainen S, Tokola H, Kerkela R, Ruskoaho H. GATA transcription factors in the developing and adult heart. Cardiovasc Res 2004;63:196-207.

22. Arnett DK, de las Fuentes L, Broeckel U. Genes for left ventricular hypertrophy. Curr Hypertens Rep 2004;6:36-41. 